\title{
Bilateral Subarachnoid Hemorrhage and bilateral Intracranial Hemorrhage during Dobutamine Stress Echocardiography
}

\author{
Hafiz Ghafoor $^{1}$, Nitish Sharma ${ }^{1}$, Somwail Rasla ${ }^{1}$, Choudhry Humayun ${ }^{2}$, and Dimitrios \\ Angelis ${ }^{1}$ \\ ${ }^{1}$ Saint Vincent Hospital at Worcester Medical Center \\ ${ }^{2}$ Affiliation not available
}

June 8, 2020

\begin{abstract}
Dobutamine stress echocardiogram (DSE) is routinely used in the clinical assessment of patients with known or suspected coronary artery disease (CAD). DSE can cause serious complications including cerebrovascular accident (CVA). Even though, the incidence of CVA associated with DSE is very low $(<0.01 \%)$, it can be life-threatening or cause significant morbidity. We present a patient who also developed acute multifocal intracranial (ICH) and subarachnoid hemorrhages (SAH) during the DSE. A 39 years old female, with no prior cardiac history, presented to the outpatient echocardiography lab for DSE. She had blunted heart rate response with increasing dose of dobutamine $30 \mu \mathrm{g} / \mathrm{kg} / \mathrm{min}$ and was given one milligram of atropine. The patient complained of frontal headache, nausea, and severe dyspnea. CT head showed acute multifocal bilateral SAH, left frontal and right parieto-occipital ICH. Hypertension is one of the risk factors for ICH and dobutamine infusion can exacerbate severe acute hypertension, which can cause acute intraparenchymal hemorrhage. Even though the risk of ICH associated with DSE is extremely low, there should be increased vigilance if there is development of severe acute hypertension and the operator should keep a low threshold for further evaluation if the patient develops neurological symptoms.
\end{abstract}

\section{LEARNING OBJECTIVES}

- Intracranial hemorrhage is an uncommon complication of dobutamine stress echocardiogram (DSE).

- Hypertension is one of the risk factors for ICH and dobutamine infusion can exacerbate severe acute hypertension

- Even though the risk of intracranial hemorrhage (ICH) associated with DSE is extremely low, there should be increased vigilance if there is development of severe acute hypertension and the operator should keep a low threshold for further evaluation if the patient develops neurological symptoms.

\section{INTRODUCTION}

DSE is considered more specific to diagnose coronary artery disease (CAD) compared to other stress modalities, ${ }^{(1)}$ hence routinely used in the clinical assessment of patients with known or suspected CAD. DSE can cause serious complications including cerebrovascular accident (CVA). ${ }^{(2)}$ Even though, the incidence of CVA associated with DSE is very low $(<0.01 \%),{ }^{(2)}$ it can be life-threatening or cause significant morbidity. There is a paucity of cases of CVA during DSE. ${ }^{(3,4)}$ We present a patient who also developed acute multifocal subarachnoid hemorrhages (SAH), and ICH during the DSE. Patient was treated conservatively because she was neurologically intact and the work up showed no evident cause of bleed.

CASE REPORT

History of presentation: 
A 39 years old Hispanic female, with no prior cardiac history, presented to the outpatient echocardiography lab for DSE, as a workup for her persistent atypical chest pain on exertion. The patient had been well until one month when she started feeling substernal pressure sensation, that prompted her visit to primary care physician. She underwent an exercise stress test that was inconclusive given the non-specific electrocardiogram (ECG) changes with poor exercise capacity. Her persistent atypical chest pain promoted her primary care provider to refer her for pharmacological stress test.

Prior to the day of the test, the patient complained of a nonspecific frontal headache that ultimately resolved with one dose of Tylenol $350 \mathrm{mg}$. Vitals signs before the stress test were: resting heart rate (HR) - 66 beats per minute (bpm), and blood pressure (BP) - 150/70 $\mathrm{mmHg}$, the respiratory rate 22 breaths per minute, and the oxygen saturation $100 \%$ while she was breathing ambient air. As per protocol, dobutamine infusion was initiated at $10 \mu \mathrm{g} / \mathrm{kg} / \mathrm{min}$, subsequently increased by $10 \mu \mathrm{g} / \mathrm{kg} / \mathrm{min}$ at $3-\mathrm{min}$ intervals. She had blunted heart rate response with increasing dose of dobutamine and vitals at $30 \mu \mathrm{g} / \mathrm{kg} / \mathrm{min}$ were $\mathrm{HR} 78 \mathrm{bpm}$ and BP 161/88 mmHg. One milligram of atropine was given to get the desired response, and her HR increased to $175 \mathrm{bpm}$ and BP to $240 / 150 \mathrm{mmHg}$. The infusion was stopped immediately due to the hypertensive response. Images were obtained at peak heart rate and in recovery.

The patient complained of frontal headache associated with nausea, worse than what she had in the days before the test. She also started to complain of severe dyspnea with increased oxygen requirement up to 5 liters per nasal cannula. The patient was given sublingual nitro $0.4 \mathrm{mg}$ and two doses of metoprolol 5 mg intravenous and promptly transferred to the emergency department. CT head showed acute multifocal bilateral SAH, left frontal and right parieto-occipital ICH without midline shift. She was admitted to intensive care for ICH management. Her chest X-ray showed significant pulmonary edema.

Past Medical History: Obesity

Investigations:

- Non-contrast CT head revealed acute multifocal bilateral SAH, left frontal and right parieto-occipital ICH without midline shift (Figure 1 ).

- CT angiogram of head and neck followed by catheter guided cerebral angiography showed no aneurysm, arteriovenous malformation (AVM); distal left middle cerebral artery LMCA branches showed some narrowing which improved with intra-arterial Verapamil.

- DSE findings suggestive of severe global hypokinesis (Video 1, Video 2 ).

- Transthoracic Echocardiogram (TTE) showed resolution of apical hypokinesis (Video 3 ).

Management:

The patient was evaluated by Neurology and Neurosurgery and decision was made to treat conservatively since there were no significant neurological symptoms and there was no aneurysm, AVM or active bleed during cerebral angiography. Also, the distal LMCA branches showed some narrowing which improved with intra-arterial Verapamil suggestive of Reversible cerebral vascular syndrome (RCVS).

Follow up:

The patient remained free from symptoms, repeat CT head showed resolution of the bleed. She was started on Verapamil $80 \mathrm{mg}$ TID by her primary neurologist with no further episodes of headache.

\section{DISCUSSION}

Indication for DSE includes diagnosis of CAD, risk stratification of patients with chronic CAD, acute or chronic myocardial infarction (MI), or valvular heart disease. DSE has been known to cause serious complications even though it is considered a safe stress modality. These severe complications include myocardial ischemia, myocardial infarction, atrial and ventricular arrhythmias, hypertension, hypotension, myocardial rupture, and CVA. (2)

Hypertension is one of the risk factors for ICH. ${ }^{(5)} \mathrm{ICH}$ associated with hypertension is known to involve small 
penetrator arteries ${ }^{5}$. Dobutamine infusion can exacerbate severe acute hypertension, which can cause acute intraparenchymal hemorrhage. First reported case of ICH associated with DSE was a patient on chronic anticoagulation for a prosthetic mitral valve. (4) In the second case, ICH occurred immediately following DSE that resulted in uncal herniation in the absence of anticoagulation, ${ }^{(3)}$ but all cases had an exaggerated hypertensive response to Dobutamine. Furthermore, in a large registry on the safety of DSE, only 3 patients suffered a stroke, but the mechanism of stroke was not reported. (9)

In a systematic review, DSE has been shown to induce transient cardiomyopathies including stress cardiomyopathy with apical ballooning in $95 \%$ of the study patients. ${ }^{(6)}$ Various mechanisms include catecholamine excess have been postulated for wall motion abnormalities in cardiomyopathies. Cardiomyopathy appears to be associated with several acute neurological diseases like SAH. Stroke is linked to cardiomyopathies by

a dual relationship since it may induce cardiomyopathy by catecholamine release and cardiomyopathy itself may be complicated by left ventricular thrombi leading to stroke. ${ }^{(8)}$

In addition to ICH, our patient had features suggestive of cardiomyopathy based on the DSE findings of hypokinesis of distal anterior, the apical and distal septal wall at peak stress with possible augmentation of the base and her post stress images reveal LV global hypokinesis. (Video 1, Video 2 ). However, the subsequent repeat TTE showed resolution of global hypokinesis. (Video 3 ).

Our patient developed ICH due to the exaggerated hypertensive response to Dobutamine and was diagnosed with Reversible cerebral vasoconstriction stroke (RCVS) after the vaso-reactive testing was done. Calcium channel blockers are shown to be beneficial for the management of the vasospasm.

\section{Conclusion:}

The significance of this case, like the others, lies in advising caution for patients undergoing DSE with SBP $>180 \mathrm{mmHg}$ during the procedure and HR exceeding $85 \%$ of the maximal predicted for age. Caution should be taken even if this effect was transient in otherwise healthy young patient as illustrated in this case. The ACC/AHA statement recommends terminating a treadmill test for an $\mathrm{SBP}>250 \mathrm{~mm} \mathrm{Hg}$ or $\mathrm{DBP}>115 \mathrm{~mm}$ $\mathrm{Hg}$ during the test.

\section{CONFLICT OF INTEREST}

We wish to confirm that there are no known conflicts of interest associated with this publication and there has been no significant financial support for this work that could have influenced its outcome.

We confirm that the manuscript has been read and approved by all named authors and that there are no other persons who satisfied the criteria for authorship but are not listed. We further confirm that the order of authors listed in the manuscript has been approved by all of us.

We confirm that we have given due consideration to the protection of intellectual property associated with this work and that there are no impediments to publication, including the timing of publication, with respect to intellectual property. In so doing we confirm that we have followed the regulations of our institutions concerning intellectual property.

Hafiz U. Ghafoor, on behalf of all co-authors

\section{REFERENCES}

1. Cheitlin MD, Armstrong WF, Aurigemma GP, Beller GA, Bierman FZ, Davis JL, Douglas PS, Faxon DP, Gillam LD, Kimball TR, Kussmaul WG, Pearlman AS, Philbrick JT, Rakowski H, Thys DM et al. ACC/AHA/ASE 2003 guideline update for the clinical application of echocardiography-summary article: a report of the American College of Cardiology/American Heart Association Task Force on 
Practice Guidelines ACC/AHA/ASE Committee to Update the 1997 Guidelines for the Clinical Application of Echocardiography). J Am Coll Cardiol 2003;42:954-70.

2. Geleijnse M, Krenning B, Nemes A, van Dalen BM, Soliman OI, ten Cate FJ, Schinkel AF, Boersma E, Simoons ML. Incidence, pathophysiology, and treatment of complications during dobutamine-atropine stress echocardiography. Circulation 2010;121:1756-67.

3. Bennin C, Ramoutar V, Velarde G. Intraparenchymal hemorrhage and uncal herniation resulting from dobutamine stress echocardiography. BMJ Case Rep. 2014 Mar 18;2014. pii: bcr2013201891. doi: 10.1136/bcr-2013-201891.

4. Shah SP, Chaudhry GM. Intracranial hemorrhage as a complication of dobutamine stress echocardiography: case report and review of the literature. Echocardiography. 2012 May;29(5):E119-21. doi: 10.1111/j.1540-8175.2011.01623.x.

5. Thrift AG, McNeil JJ, Forbes A, Donnan GA. Three important subgroups of hypertensive persons at greater risk of intracerebral hemorrhage. Melbourne Risk Factor Study Group. Hypertension 1998;31:1223-9.

6. Chandraprakasam S, Alla VM, Mooss AN, Hunter CB. Dobutamine stress testing induced transient cardiomyopathy: A systematic review. Int J Cardiol Heart Vessel. 2014 Aug 8;4:221-222. doi: 10.1016/j.ijchv.2014.07.005. eCollection 2014 Sep.

7. Merli E., Sutcliffe S., Gori M., Sutherland G. Tako-Tsubo cardiomyopathy: new insights into the possible underlying pathophysiology. Eur J Echocardiogr. 2006;7(1):53-61.

8. Ranieri M, Finsterer J, Bedini G, Parati EA, Bersano A. Takotsubo Syndrome: Clinical Features, Pathogenesis, Treatment, and Relationship with Cerebrovascular Diseases. Curr Neurol Neurosci Rep. 2018 Mar 22;18(5):20. doi: 10.1007/s11910-018-0833-7.

9. Varga A, Garcia MAR, Picano E. Safety of stress echocardiography (from the international stress echo compilation registry). Am J Cardiol. 2006 Aug 15;98(4):541-3. Epub 2006 Jun 28.

\section{FIGURE LEGEND}

1. Figure 1. CT Head demonstrating (A) left frontal intraparenchymal, (B) right parieto-occipital intraparenchymal hemorrhage and (C) multifocal bilateral subarachnoid hemorrhages (SAH).

2. Video 1 . Apical 4 Chamber DSE findings of hypokinesis of distal anterior, the apical and distal septal wall at peak stress with possible augmentation of the base and her post stress images reveal LV global hypokinesis.

3. Video 2 . Parasternal Long Axis DSE findings of hypokinesis of distal anterior, the apical and distal septal wall at peak stress with possible augmentation of the base and her post stress images reveal LV global hypokinesis.

4. Video 3 . TTE showed resolution of global hypokinesis.

Figure 1 


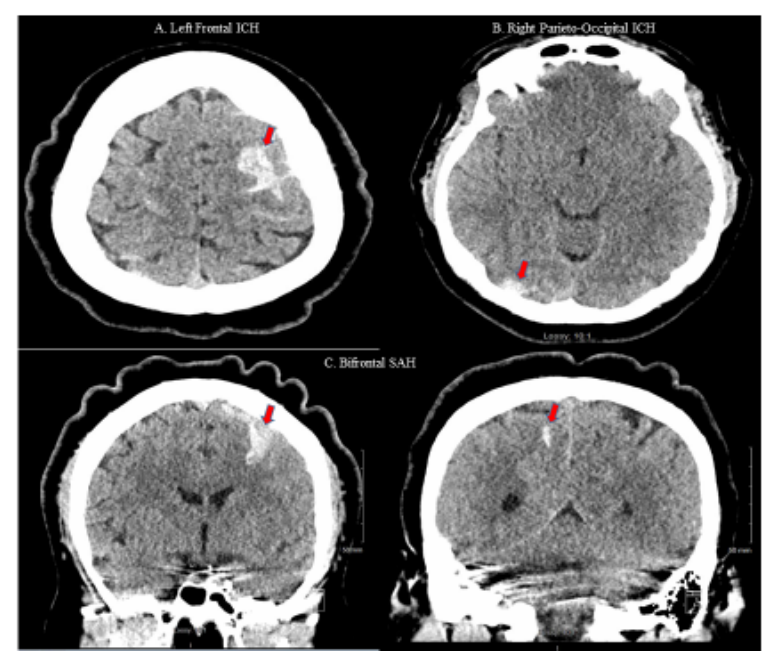

